\title{
Short-term efficacy of etamsylate injection in chronic patellar tendinopathy
}

\section{Summary}

Chronic patellar tendinopathy is one of the most common overuse knee disorders. Etamsylate injection, performed in the peritendinous tissue of chronic patellar tendinosis resulted in reduction of pain and pathologic neovascularization with improvement of leg function, after four weeks of treatment.

Keywords: patellar tendinosis, etamsylate injection, fibroblast growth factor
Volume 7 Issue 3 - 2017

Pedro Cuevas,' José Antonio Rodas, ${ }^{2}$ Javier Angulo, ${ }^{3}$ Guillermo Giménez-Gallego ${ }^{4}$

'Universidad Alfonso X el Sabio, Spain

${ }^{2}$ Mutualidad de Previsión Social de Futbolistas Españoles, Spain ${ }^{3}$ Servicio de Histología, Departamento de Investigación, Hospital Universitario Ramóny Cajal, Spain

${ }^{4}$ Departamento de Estructura y Función de Proteínas, Centro de Investigaciones Biológicas, Spain

Correspondence: Pedro Cuevas, Facultad de Medicina, Universidad Alfonso X el Sabio, 2869I-Villanueva de la Cañada, Spain, Email pedro.cuevas44@gmail.com

Received: August 24, 2017 | Published: August 29, 2017

\section{Case presentation}

A 35-old year healthy Caucasian football player man presented with a 3years history of anterior knee pain and tenderness in his right patellar tendon insertion. He referred a constant dull aching pain with walking, forcing to discontinue sport because of the severity of his pain. He has been previously treated with conservative therapy and with platelet-rich plasma (PRP) injections; however his pain did not improve. At presentation, pain was rated as 7 out of 10 on Visual Analogue Scale (VAS). Color Doppler ultrasound examination revealed signs of tendinosis with significant neovascularity, formed by immature tortuous and dilated vessels into intratendinous mass (Figure 1A). Furthermore, color Doppler scans showed areas of intratendinousedema in the proximal patellar-tendon interface (Figure 1A). After discussing the various treatment options, the patient opted to try etamsylate injection and signed an informed consent. Lidocaine was infiltrated into the skin overlying the right patellar tendon. $4 \mathrm{ml}$ etamsylate (Dicynone ${ }^{\circledR}$, Sanofi-Aventis. France) were injected under ultrasound guidance into peritendinous patellar tendon tissue. The procedure was uneventful. At 2 weeks follow-up visit, patient reported a marked improvement of his pain (VAS was rated as 2), and color Doppler ultrasound scans showed a significant reduction of neovessels and disappearance of tendinous edema (Figure 1B). Furthermore, at 4weeks tendon hypervascularity decreased (Figure 1C) and pain strongly ameliorated (VAS=1). Patient could return to his previous level of sport without any restrictions.

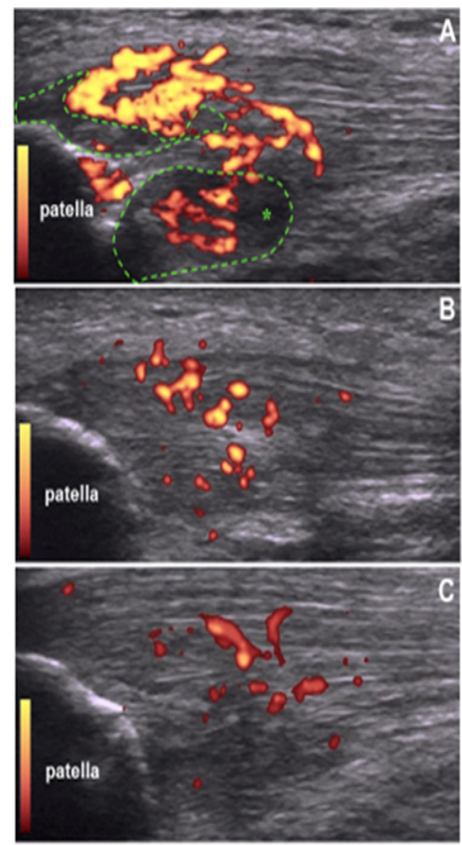

Figure I The presence of neovessels is detected in the patellar tendon using color Doppler ultrasound. Longitudinal scans taken before (A) and after two (B) and four weeks $(\mathrm{C})$ of etamsylate injection. Diminution of hypervascularity is demonstrated after injection of etamsylate. Note the reduction of edema $(*)$ after two weeks of treatment. 


\section{Discussion}

Patellar tendinosis is a common painful difficult-to-treat overuse injury of the patellar tendon with a negative impact on the carriers of many athletes. The overall prevalence of patellar tendinopathy yields to $14 \%$ in elite athletes and increases up to $45 \%$ in high-risk sports such as basketball and volleyball. ${ }^{1}$ There are a wide variety of treatment options available, the majority of which are non-operative. No consensus exists on the optimal method of treatment. Antiinflammatories and injectable agents have shown mixed results. ${ }^{2}$ In approximately $10 \%$ of conservatively treated patients, conservative treatment fails and surgery is required. ${ }^{3}$ Since tendinopathy appears to be a highly active process of ongoing neovascularization, anti-angiogenic therapies could be a new approach for treating tendinopathies. ${ }^{4}$ It has been reported that nerves appear to travel in close association to tendon neovessels. ${ }^{5}$ This finding suggests that angiogenesis plays an important role in pain-experienced in tendinopathy condition. ${ }^{6,7}$ These data are in accordance with clinical studies showing that strategies to destroy neovessels (i.e local injection of a sclerosing agent, polidocanol) lead to pain improvement. ${ }^{7}$ Etamsylate, an inhibitor of fibroblast growth factor (FGF), when locally applied, supresses FGF-driven angiogenesis. ${ }^{8,9}$ Since FGF participates in nociception, ${ }^{10,11}$ injecting etamsylate into the areas of tendon neovascularization could not only decrease the number of pathologic neovessels, but also eradicate the pain-generation nerve fibres. In conclusion, therapeutic modulation of neovascularization by influencing the levels of FGF might be a promising target for new approaches in tendinopathies.

\section{Learning points}

i. Neovascularization has been demonstrated in painful tendon from sport activity.

ii. Hypervascularity is one of the causes of tendon pain due to parallel migration of neovascular innervation.

iii. Etamsylate injection in pathologic patellar tendon resulted in clinical improvement.

iv. Etamsylate, acting as an FGF inhibitor, could destroy pathological tendon neovessels and decrease the nociceptive FGF-related activities.

\section{Acknowledgements}

None.

\section{Conflict of interest}

The author declares no conflict of interest.

\section{References}

1. Lian OB, Engebretsen L, Bahr R. Prevalence of jumper's knee among elite athletes from different sports:a cross-sectional study. Am J Sports Med. 2005;33(4):561-567.

2. Andres BM, Murrell GA. Treatment of tendinopathy: what works, what does not, and what is on the horizon. Clin Orthop Relat Res. 2008;466(7):1539-1554.

3. Brockmeyer M, Haupert A, Kohn D, et al. Surgical Technique: Jumper's knee-arthroscopic treatment of chronic tendinosis of the patellar tendon. Arthrosc Tech. 2016;5(6):e1419-e1424.

4. Pufe T, Petersen WJ, Mentlein R, et al. The role of vasculature and angiogenesis for the pathogenesis of degenerative tendons disease. Scand J Med Sci Sports. 2005;15(4):211-222.

5. Hoksrud A, Torgalsen T, Harstad H, et al. Ultrasound-guided sclerosis of neovessels in patellar tendinopathy: a prospective study of 101 patients. Am J Sports Med. 2012;40(3):542-547.

6. Knobloch K, Kraemer R, Jagodzinski M, et al. Eccentric training decreases paratendon capillary blood flow and preserves paratendon oxygen saturation in chronic achilles tendinopathy. J Orthop Sports Phys Ther. 2007;37(5):269-276.

7. Cook JL, Malliaras P, De Luca J, et al. Neovascularization and pain in abnormal patellar tendons of active jumping athletes. Clin J Sport Med. 2004;14(5):296-299.

8. Fernández IS, Cuevas P, Angulo J, et al. Gentisic acid, a compound associated with plant defense and a metabolite of aspirin, heads a new class of in vivo fibroblast growth factor inhibitors. $J$ Biol Chem. 2010;285(15):11714-11729.

9. Angulo J, Cuevas P, Cuevas B, et al. Diacetyloxyl derivatization of the fibroblast growth factor inhibitor dobesilate enhances its anti-inflammatory, anti-angiogenic and anti-tumoral activities. $J$ Transl Med. $2015 ; 13: 48$

10. Andres C, Hasenauer J, Ahn HS, et al. Wound-healing growth factor, basic FGF, induces Erk1/2-dependent mechanical hyperalgesia. Pain. 2013;154(10):2216-2226.

11. Liu H, Wu QF, Li JY, et al. Fibroblast growth factor 7 is a nociceptive modulator secreted via large dense-core vesicles. $J$ Mol Cell Biol. 2015;7(5):466-475. 